PALEO

Revue d'archéologie préhistorique

21 | 2009-2010

Varia

\title{
Le plus ancien enfant d'Aquitaine : Combe-Grenal 31 (Domme, France)
}

Bruno Maureille, María Dolores Garralda, Stéphane Madelaine, Alain Turq et Bernard Vandermeersch

\section{(2) OpenEdition}

Journals

Édition électronique

URL : http://journals.openedition.org/paleo/1814

DOI : $10.4000 /$ paleo.1814

ISSN : 2101-0420

Éditeur

SAMRA

Édition imprimée

Date de publication : 1 mars 2011

Pagination : 189-202

ISSN : 1145-3370

\section{Référence électronique}

Bruno Maureille, María Dolores Garralda, Stéphane Madelaine, Alain Turq et Bernard Vandermeersch,

"Le plus ancien enfant d'Aquitaine : Combe-Grenal 31 (Domme, France) », PALEO [En ligne], 21 |

2009-2010, mis en ligne le 21 octobre 2011, consulté le 07 juillet 2020. URL : http://

journals.openedition.org/paleo/1814; DOI : https://doi.org/10.4000/paleo.1814

\section{(c) $(1)$}

PALEO est mis à disposition selon les termes de la licence Creative Commons Attribution - Pas d'Utilisation Commerciale - Pas de Modification 4.0 International. 


\title{
LE PLUS ANCIEN ENFANT D'AQUITAINE : Combe-Grenal 31 (Domme, France)
}

\author{
Bruno MAUREILLE ${ }^{(1)}$, María Dolores GARRALDA ${ }^{(2,1)}$, \\ Stéphane MADELAINE ${ }^{(3,4)}$, Alain TURQ ${ }^{(3,4)}$ et Bernard VANDERMEERSCH ${ }^{(1)}$
}

Résumé : Le récolement des collections du Musée national de Préhistoire a permis, lors du travail sur la faune découverte au cours des fouilles de F. Bordes à Combe-Grenal, site de référence, l'identification d'un nouveau fossile humain, Combe-Grenal 31. II provient de la couche 60. En fonction des vestiges archéologiques et paléontologiques qu'elle livre, et de comparaisons avec des niveaux aussi anciens de différents gisements, cette couche s'est probablement formée lors du dernier tiers du stade isotopique 6.

Combe-Grenal 31 correspond à une incisive inférieure droite de la dentition déciduale d'un enfant d'environ 3 ans \pm 12 mois. Sa couronne présente de grandes dimensions malgré une forte attrition de la face occlusale. La courbure de la face vestibulaire, ainsi que le tubercule lingual sont bien marqués. Les crêtes marginales sont un peu saillantes. Ses caractères morphologiques et leur comparaison avec d'autres fossiles européens ainsi que l'ellipse d'équiprobabilité réalisée à partir des dimensions de la couronne nous permettent de souligner des similitudes avec les dents équivalentes d'autres enfants néandertaliens des stades isotopiques 5,4 ou 3 et deux spécimens européens rapportés au stade 6 .

Mots-clés : Combe-Grenal, Néandertal, dent déciduale, incisive, stade isotopique 6.

Key-words: Combe-Grenal, Neandertal, deciduous tooth, incisor, isotopic stage 6.

\section{Abridged English version}

The earliest Aquitanian child: Combe-Grenal 31 (Domme, France).

\section{Introduction}

The important archaeological site of Combe-Grenal is located (fig. 1) near the medieval city of Domme and the Dordogne valley, in the south-west of France. It is a rock-shelter, used as an habitation site by different human groups in the course of time.

Although the site had been known since the $19^{\text {th }}$ century, the first small archaeological excavations were undertaken by $D$. and E. Peyrony, around 1930. However, the most significant fieldwork at the site was carried out by F. Bordes between 1953 and 1965 (Bordes 1955, 1966, 1971, 1972). This work showed that Combe-Grenal possessed a complex stratigraphy, over 13m thick, covering the period between the isotopic stages 6 and 3 corresponding culturally to the late or " meridional Acheulian " (levels 64 to 56) and the Mousterian (levels 55 to 1).

(1) UMR PACEA, Laboratoire d'Anthropologie des Populations du Passé, Université de Bordeaux, avenue des Facultés, Université Bordeaux 1, 33405 Talence cedex - b.maureille@anthropologie.u-bordeaux1.fr ; bvanderm@bio.ucm.es

(2) U. D. de Antropología Física, Facultad de Biología, Universidad Complutense de Madrid, Ciudad Universitaria, E-28040 Madrid mdgarra@@bio.ucm.es; bvanderm@bio.ucm.es

(3) Musée national de Préhistoire, 1 rue du Musée, 24620 Les Eyzies-de-Tayac - stephane.madelaine@culture.gouv.fr ; alain.turq@culture.gouv.fr

(4) UMR PACEA, Institut de Préhistoire et de Géologie du Quaternaire, Université de Bordeaux, avenue des Facultés, Université Bordeaux 1, 33405 Talence cedex. 
Numerous studies, in addition to the ones cited above, about faunal, geological and archaeological aspects (Bordes et al. 1966 ; Bordes et al. 1972; Laville 1969a and b, 1975 ; Laville et al. 1984 ; Paquereau 1970, 1974-75 ; Levine 1983 ; Chase 1983, 1986a and b, 1989 ; Lenoir 1986 ; Panabières 1986 ; Guadelli and Laville 1990 ; Delpech and Prat 1995 ; Bourguignon and Turq 2003 ; Steele 2003, 2004 ; Fernandez et al. 2006 ; Monnier 2006 ; Thiébaut 2006 ; Hiscock and Clarkson 2007 ; Faivre 2008 ; Dibble et al. 2009) have provided a huge amount of highly interesting information about this site.

\section{Combe-Grenal human remains}

Several human remains have also been found at Combe-Grenal; four by D. and E. Peyrony's excavation, and the rest by F. Bordes. Although some of the latter remains had been published by Genet-Varcin (1982), the assemblage was studied and described in detail, from the taphonomic, morphological and pathological points of view, by Garralda and Vandermeersch (1997, 2000a and b, 2007). The most important of these studies (Garralda and Vandermeersch 2000a) describes an incomplete tooth from Level 39, a frontal fragment from Level 35, and 24 fossils (teeth and different parts of the cranial and postcranial skeleton) found in Level 25 (as well as the other four remains recovered by D. and E. Peyrony). Marks of manipulation had been identified on some of the fragments by Genet-Varcin (1982) and Le Mort (1989), and these were re-analysed and compared by Garralda and Vandermeersch (2000a ; Garralda et al. 2003, 2005 ; Garralda 2008).

The anthropological collection from Combe-Grenal is therefore important for the Mousterian period in SW France. Geological and faunal research carried out, above all by Guadelli and Laville (1990) have succeeded in attributing Level 39 to isotopic stage 5a, while Levels 35 and 25 correspond to isotopic stage 4. Consequently, almost all the human fossils from CombeGrenal (those from level 25) belong chronologically to the period $\sim 75 / 65 \mathrm{ky}$ BP. The palaeo-environmental data confirms that these populations lived in an intensely cold and generally dry climate.

\section{The new fossil}

In 2009, another human fossil was identified amongst the fauna from Level 60 at Combe-Grenal (fig. 2), and therefore much older than the above assemblage and corresponding to isotopic stage 6 . The industry (fig. 3) which accompanied it was called «meridional Acheulean » by F. Bordes, although modern opinion interprets it as a Proto-Mousterian or a very old Mousterian. The place of the discovery is situated much further forward from where the well-documented remains in Level 25 were found (Garralda and Vandermeersch 2000a), i.e. in the front part of the cave that existed at that time.

\section{Description}

The fossil consists of an incomplete tooth, of which much of the root is missing. Its small size and its shape (Fig. 4) allowed us to identify it as belonging to the deciduous dentition, to be precise, as the lower right central incisor of a child. The lingual tubercle is very prominent. The root was broken post-mortem, without any visible signs of reabsorption, so we can assign it to a child who died at the age of 3 years \pm 12 months, probably a little older than the child from Roc-de-Marsal 1 .

The crown size and indices are large and similar to those of the same tooth in other Neandertal children (Table 1), although the mesio-distal diameter has been reduced by the presence of inter-proximal facets. The occlusal wear is very marked.

\section{Comparisons}

As well as comparing the basic data given in Table 1, we have also carried out bivariate analysis (Table 2, Fig. 5) showing that Combe-Grenal 31 lies outside $95 \%$ of modern human variability and is situated within the range of variation for Pre-neandertal and Neandertal children.

\section{Conclusions}

Combe-Grenal 31 is a lower right deciduous central incisor, with marks of inter-proximal attrition and on the occlusal edge. Its morphology and diameters are very similar to those of other Neandertal children. No information is available to help us interpret how and why this fossil was found in Level 60, together with remains of implements and fauna. The main interest of this incomplete tooth lies from its provenance one of the deepest levels of the famous deposit of Combe-Grenal, Level 60, attributed to the isotopic stage 6 and with a lithic assemblage interpreted as Proto-Mousterian or very old Mousterian. That means that this child's tooth is the oldest human fossil in Aquitaine, a representative of the human group who occupied Combe-Grenal about 130 ky ago i. e. the last third of the isotopic stage 6 (Martrat et al. 2004, 2007). There are not many sites in the region that have yielded pene-contemporary anthropological specimens, considering that the level where CombeGrenal 31 was found is just a little more recent than that at l'Abri Suard (Charente), and nearly 70,000 years older than CombeGrenal Level 25, where so many Neandertal remains were discovered. 


\section{Introduction}

La grotte de Combe-Grenal (fig. 1) se situe dans un petit vallon sur la rive gauche de la Dordogne, à l'est du village de Domme (à 10 km au sud-est de Sarlat, en Dordogne). Connue depuis le début du XIXe siècle (Bordes 1972), elle a été visitée par divers chercheurs dont $\mathrm{E}$. Lartet et $\mathrm{H}$. Christy (1864) puis L. Capitan et D. Peyrony vers le début du XX siècle. Au début des années 1930, D. et E. Peyrony procèdent à une fouille limitée du site. Mais ce sont les travaux de F. Bordes entre 1953 et 1965 qui l'ont placée parmi les plus importants gisements français du Paléolithique moyen (Jaubert 1999) en raison de la puissance de sa séquence archéo-stratigraphique avec une importante succession d'occupations humaines (Bordes 1972 ; Faivre 2008).

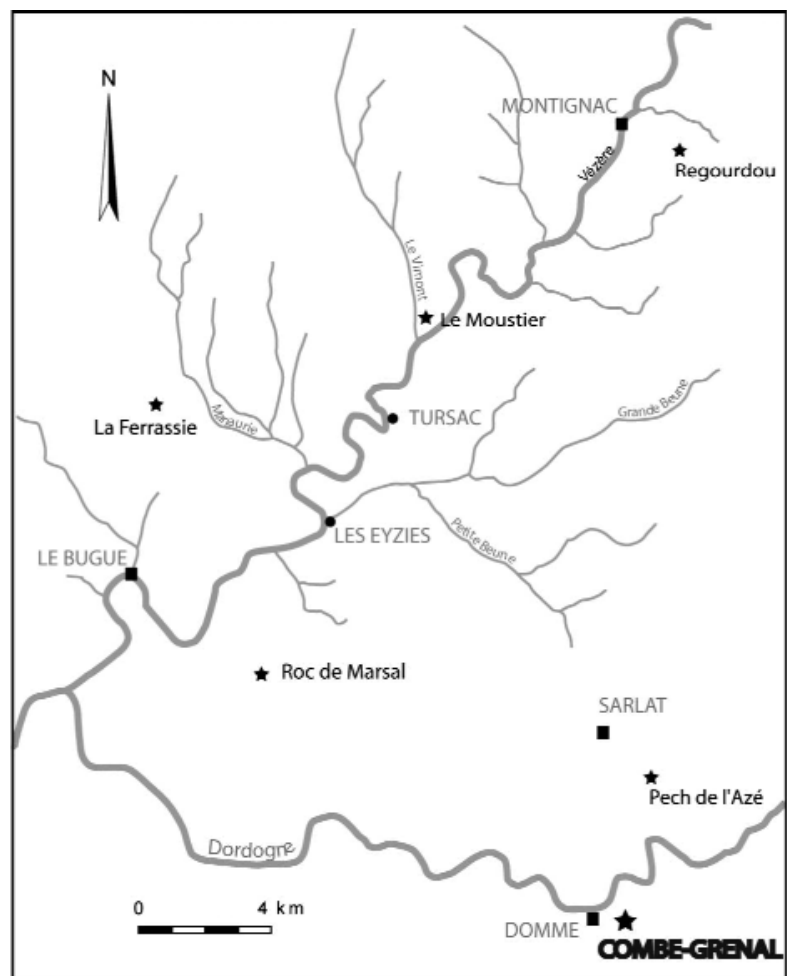

Figure 1 - Localisation du site de Combe-Grenal et d'autres gisements moustériens de Dordogne ayant livré des restes humains.

Figure 1 - Location of Combe-Grenal site and other mousterian places delivering hominin remains.

François Bordes a réalisé de nombreuses découvertes archéologiques à Combe-Grenal qu'il a décrites dans plusieurs travaux (Bordes 1955, 1966, 1971 ; Bordes et Prat 1965 ; Bordes et al. 1966 ; Bordes et al. 1972) et spécialement dans son ouvrage publié en 1972. Des dépôts sédimentaires et archéologiques forment une séquence de plus de 13 mètres d'épaisseur mise en place lors du stade isotopique 6 et s'achevant lors du stade isotopique 3 . François Bordes les subdivisa en 64 couches (65, puisque la couche 50 a été divisée en 50 et 50A). II indique (Bordes 1972) qu'au sein de cette séquence qui, selon la sédimentologie et la palynologie, se serait formée au cours de 22 phases climatiques, peuvent être distinguées trois unités principales :

- couches 64 à 56 : « industrie acheuléenne ». Cet ensemble se serait formé vers la fin du stade isotopique 6, comme nous le verrons plus loin. L'interprétation de l'assemblage lithique est aujourd'hui modifiée ;

- couches 55 à 36 : leur dépôt serait contemporain des manifestations de 7 phases climatiques selon les travaux de sédimentologie (Laville 1975). Toutes livrent du Moustérien typique sauf la couche 38 qui livre du Moustérien à denticulés. Elles se seraient formées lors du stade isotopique 5 ;

- de la couche 35 à la 1 : selon H. Laville (1975), huit phases climatiques se seraient succédées. A la base de la séquence, les conditions sont très froides et peu humides (stade isotopique 4). Elles évolueraient vers des conditions moins rigoureuses pour être relativement douces au sommet de la séquence (stade 3 ). Différents faciès du Moustérien y ont été identifiés, notamment du Moustérien de type Quina.

De nombreux autres travaux archéologiques, géologiques, palynologiques et paléontologiques ont été réalisés sur l'importante masse de données fournies par ce gisement (Laville 1969a et b, 1975 ; Paquereau 1970, 1974-75 ; Laquay 1981 ; Donard 1982 ; Chase 1983, 1986a et b, 1989 ; Levine 1983 ; Laville et al. 1984 ; Lenoir 1986 ; Panabières 1986 ; Guadelli 1987 ; Marquet 1989 ; Guadelli et Laville 1990 ; Turq 1992 ; Delpech et Prat 1995 ; Delpech et al. 1995 ; Bourguignon et Turq 2003 ; Steele 2003, 2004 ; Turq 2003 ; Fernandez et al. 2006 ; Monnier 2006 ; Thiébaut 2006 ; Hiscock et Clarkson 2007 ; Faivre 2008). Le plus récent (Dibble et al. 2009) est l'analyse de la répartition spatiale des vestiges archéologiques cotés de six niveaux, à partir des données de F. Bordes et de l'état actuel de la collection. II met en évidence des problèmes stratigraphiques dont l'importance reste à déterminer, problèmes qui ne modifient toutefois pas la succession des couches et l'intégrité des ensembles culturels.

\section{1 - Les restes humains mis au jour dans le gisement}

Plusieurs restes humains ont été mis au jour à CombeGrenal, quatre durant les fouilles de D. et E. Peyrony et les autres, pendant celles dirigées par F. Bordes. Certains de ces derniers ont été publiés par Genet-Varcin (1982) et l'ensemble a été analysé en détail, tant du point de vue taphonomique que morphologique ou pathologique, par Garralda et Vandermeersch (1997, 2000a et b, 2007). Ainsi, une dent incomplète de la couche 39 , un fragment de frontal de la couche 35 , et 24 autres pièces (dents et différentes parties du squelette crânien et infra-crânien) découvertes dans la couche 25 (comme les 4 pièces des fouilles Peyrony) ont donc été très précisément étudiés. De plus, rappelons que des traces de manipulations anthropiques 
apparaissant sur quelques fragments ont été d'abord décrites par Genet-Varcin (1982) puis Le Mort (1989). Elles ont été analysées à nouveau par Garralda et Vandermeersch (2000a), Garralda et al. (2003 et 2005), Garralda 2008.

La série anthropologique de Combe-Grenal est donc très importante pour la compréhension de la variabilité des Moustériens du sud-ouest de la France, surtout pour la période allant d'environ 75-65 ka (stade isotopique 4) dans laquelle, d'après la synthèse de Guadelli et Laville (1990), se situerait le niveau 25.

\section{2- Le nouveau fossile : Combe-Grenal Hominid 31}

En juin 2009, durant les travaux de récolement des collections du Musée national de Préhistoire, l'un d'entre nous (S.M.) isola dans les collections de la faune de CombeGrenal, et précisément celle de la couche 60 , une nouvelle pièce qu'il considéra comme humaine : une incisive déciduale. La diagnose fut confirmée par B. M. qui détermina une incisive déciduale inférieure centrale.

Le fossile portait, avant la restauration (fig. 2) l'inscription " C - - / A5 / C60 », indiquant qu'il avait été mis au jour dans une des plus anciennes couches du site, la 60 (aussi dénommée A5 au début des fouilles de Bordes). Cette couche affleurait au sud et se développait bien au-dessous de la couche 25 qui a fourni l'essentiel des vestiges humains (surtout les carrés E5 et F4 ; voir Garralda et Vandermeersch 2000a).

En fonction de l'inventaire publié des vestiges humains de Combe-Grenal (Garralda et Vandermeersch, ibidem, p. 215-216), ce nouveau fossile peut être numéroté Combe-Grenal Hominid 31.

\section{Contexte « culturel » : le techno-complexe lithique}

Selon F. Bordes (1966 et 1971), l'industrie lithique que livre la couche 60 correspond à de l'Acheuléen final ou "Acheuléen méridional » en raison de la présence de bifaces et dans la mesure où ces derniers sont différents de ceux de l'Acheuléen du nord de la France (fig. 3).

Ce faciès est connu en grottes et abris à Combe-Grenal et au Pech-de-l'Azé II (couches 8 et 9 par exemple), mais aussi en plein air, en Chalosse (Thibault 1970) et en Bergeracois, à Cantalouette (Guichard 1965), Les Pendus et Barbas (Guichard J. et G. 1966). D'un point de vue typologique, il se définit principalement par un outillage bifacial grossier, souvent à pointe mousse, nucléiforme, rarement symétrique. L'outillage sur éclats est par contre très diversifié et moustérien. L'approche technologique menée par E. Boëda (1991 - p. 49-50) sur les couches 8 et 9 du Pechde-l'Azé II (fouilles F. Bordes) et C'4 de Barbas a montré la présence d'un système de production original, le « débi-

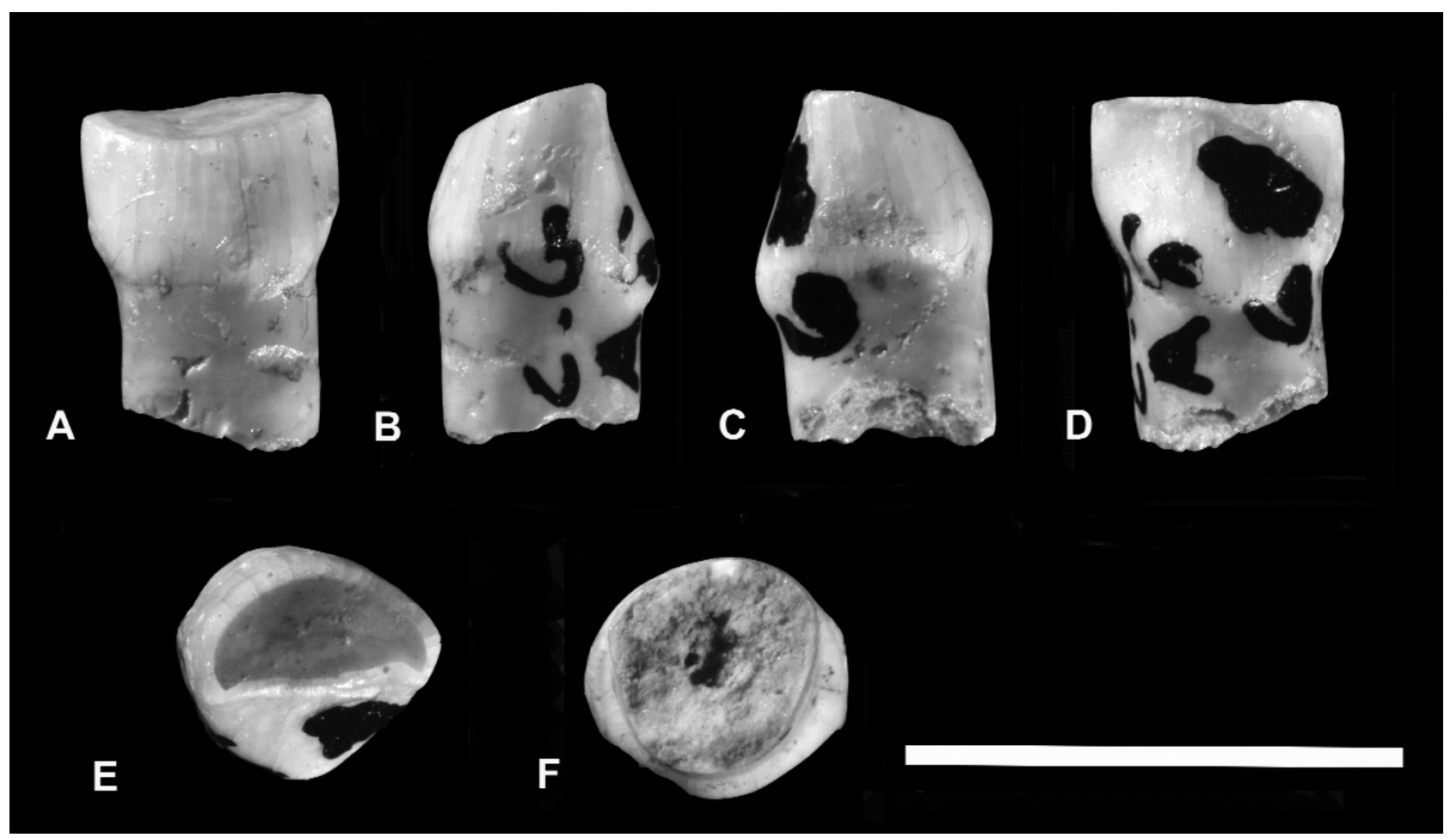

Figure 2 - Combe-Grenal 31 avant la restauration (cliché Ph. Jugie, Musée national de Préhistoire).

Figure 2 - Combe-Grenal 31 before restored (photo Ph. Jugie, Musée national de Préhistoire). 
tage trifacial " qui a fourni les supports, une partie des nucléus ayant été aménagée en bifaces très partiels.

Le réexamen de la couche 59 de Combe-Grenal (Turq 1992) ainsi que la découverte, la fouille et l'étude du site de plein air de Combe-Brune 3 (Brenet et al. 2008) ont apporté des précisions. Aujourd'hui, ce faciès se définit par :

- une exploitation des matières premières lithiques provenant de l'environnement immédiat du site ;

- des supports peu standardisés, produits par des séries courtes et unipolaires qui peuvent intéresser deux surfaces contiguës et sécantes, alternativement plan de frappe ou surface de débitage. Les nucléus sont généralement de type acheuléen (Bordes 1961 ; Rolland 1986) mais il existe aussi des nucléus à orientation centripète à une ou deux surfaces préférentielles de débitage et des nucléus trifaciaux très minoritaires ;

- le façonnage est particulier, à la fois différent de celui observé dans l'Acheuléen classique et de celui du Moustérien de tradition acheuléenne. Les bifaces vraiment symétriques sont très rares. Les autres pièces ont vu se succéder, dans un sens ou dans l'autre, des phases de production (notion de nucléus) et de façonnage (biface).

II ne s'agit pas de nucléus présentant quelques retouches localisées (vision typologique) mais des pièces reconfigurées qui changent de statut. Certains bifaces ont été défigurés ou réaménagés par un grand enlèvement, sorte de coup de tranchet obtenu au percuteur dur.

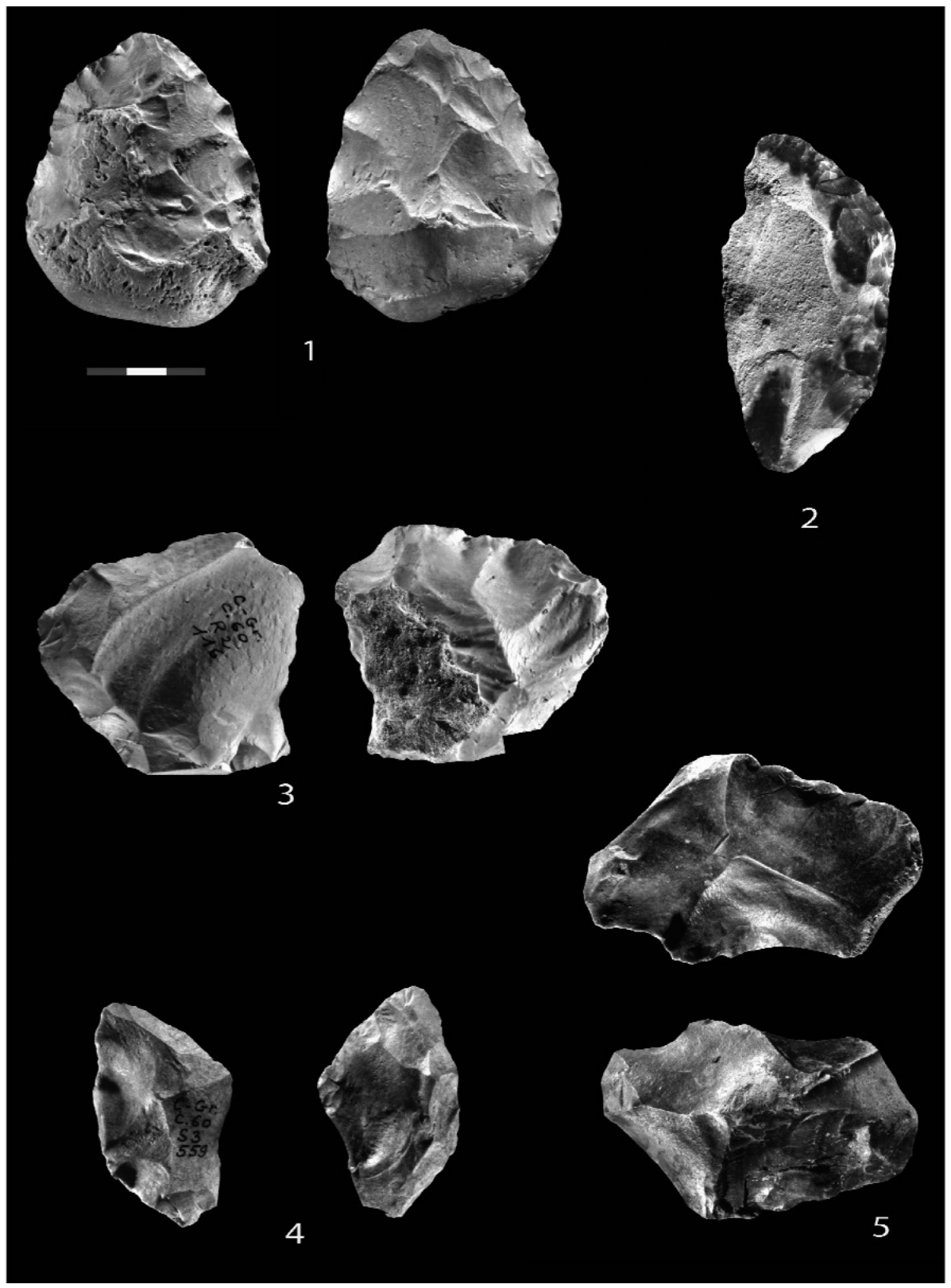

Figure 3 - Quelques objets lithiques de la couche 60 de Combe-Grenal. 1, biface symétrique à base réservée ; 2, racloir simple convexe ; 3 , éclat de réaménagement de la pointe d'un biface, le bulbe bien marqué (photo de gauche) correspond à l'utilisation d'un percuteur de pierre ; 4, nucléus dont une extrémité a été aménagée par retouche (biface-nucléus) ; 5 , nucléus à une surface préférentielle de débitage (cliché $P h$. Jugie, Musée national de Préhistoire).

Figure 3 - Some lithic tools from Combe-Grenal level 60. 1, symetric handaxe with a cortical butt ; 2 , convex simple scraper ; 3 , refitting flake of an handaxe point, the well marked bulb (left photo) corresponds to the utilization of a hard hammer ; 4 , nucleus whose extremity was fitted out by retouch (handaxe-nucleus) ; 5, nucleus with one preferential surface of debitage (photo Ph. Jugie, Musée national de Préhistoire). 
Ce techno-complexe qualifié d'“ Acheuléen méridional » n'a donc rien à voir avec l'Acheuléen (morphologie des bifaces, absence de production systématique de grands supports, outillage sur éclats très diversifié) mais fait partie intégrante de la diversité du Paléolithique moyen ancien auquel il doit être rattaché.

D'un point de vue chronologique, les données disponibles permettent de le situer avant le stade isotopique 5 (Vieillevigne et al. 2008). Cette attribution est confirmée par les datations absolues obtenues sur du matériel archéologique provenant de deux sites : Pech-de-l'Azé II (couche $8: 149 \pm 12$ à $201 \pm 16$ ka (ESR), couche $9: 117 \pm 13$ à $205 \pm 26$ ka (ESR) ; Grün et al. 1991) et Combe-Brune 3 $(156,4 \pm 11,7$ ka (TL) ; Brenet et al. 2008 ; Guibert et al. 2008). Notons que deux résultats TL sur un silex (échantilIon 601 ; Bowman et Sieveking 1983) de la couche 60 de Combe-Grenal donnent les dates de $105 \pm 14$ à $113 \pm 13$ ka. Toutefois, Guibert et al. (2008 - p. 20) considèrent que l'indice de qualité de ces résultats est de 1 ce qui signifie que " seul l'ordre de grandeur de la datation pourrait être retenu » et que l'incertitude et l'évolution des méthodes empêchent maintenant une "prise en compte suffisamment sûre des résultats ".

\section{Contexte environnemental et biochronologique}

Les faunes des niveaux les plus anciens de Combe-Grenal ont déjà fait l'objet de publications détaillant, entre autres, le contenu des vestiges osseux des mammifères de la couche 60 (Bordes et Prat 1965 ; Delpech et Prat 1980, 1995 ; Delpech et al. 1995).

Les renseignements d'ordre biostratigraphique qui en découlent sont résumés brièvement ci-dessous :

- la très large prédominance du Renne, la présence de caprinés montagnards (Chamois, Bouquetin, Thar) et d'espèces steppiques que sont le Cheval et le Bison, sont autant d'arguments plaidant en faveur de conditions climatiques rigoureuses ;

- la sous-espèce caballine représentée par Equus caballus piveteaui, l'arrivée du Bouquetin, la persistance du Thar, la présence d'un Cerf de grande taille antérieurement à l'apparition de Cervus simplicidens (à partir de la couche 59), petit Cerf qui se développera surtout au stade isotopique 5 (Guadelli 1996), la présence de l'Antilope saïga dans les couches 59 et 58 , témoignage de son incursion à la fin du stade isotopique 6 dans le sud-ouest de la France, sont autant d'éléments qui confèrent à la couche 60 son appartenance au stade 6 (ancien « Riss III »; Martrat et al. 2004, 2007). Une étude biostratigraphique fondée sur les grands mammifères (Delpech et al. 1995) avait conduit à placer l'ensemble des couches 64 à 56 de Combe-Grenal dans la même biozone que les couches IV de Vaufrey (datée de $120 \pm 10$ ka (TL) et X à III (fouilles P. David) ou 53 à 49 (fouilles A. Debénath) de l'Abri Suard (La Chaise-de-Vouthon, Charente), la couche 51 ayant été datée de 126 \pm 15 ka (Blackwell et al. 1983) ce qui conduisait à placer la période de formation des dépôts constituant cette biozone dans la deuxième moitié du stade isotopique 6 . En outre, une étude menée par F. Delpech et F. Prat (1995) a apporté des précisions sur la période de formation de l'ensemble 64 à 56 de Combe-Grenal : elle aurait été relativement brève (peut-être moins de 1000 ans), serait plus récente que celle des couches 53 à 49 de la grotte Suard, et se placerait très près de la fin du stade isotopique 6 . II en résulte que cet ensemble de Combe-Grenal, incluant la couche 60 qui nous intéresse ici, a dû se mettre en place aux alentours de 130000 ans BP.

\section{Description de la dent}

\section{Identification}

La morphologie et les petites dimensions de la couronne et de la racine de Combe-Grenal 31 nous assurent qu'il s'agit d'une dent déciduale. La couronne (fig. 4), très symétrique tant selon la face vestibulaire que la linguale, et les angles droits des côtés mésial et distal par rapport au collet, permettent de l'identifier comme une incisive centrale. II s'agit probablement d'une dent droite, en raison de la très légère déviation, vers le côté mésial, de la ligne cervicale. La morphologie de cette dent, Combe-Grenal 31, est très similaire à celle des dents équivalentes des enfants de Pech-del'Azé 1 ou Roc-de-Marsal 1, exception faite de l'attrition, beaucoup plus accentuée sur le fossile étudié.

\section{Dimensions}

La dent a été mesurée trois fois et c'est la moyenne des trois mesures qui est reportée ici. Les diamètres ont été obtenus selon la méthode de Martin (Martin et Saller 1957; Monge et al. 2005).

Ces diamètres sont un peu altérés par l'usure interproximale mais les valeurs obtenues sont très similaires à celles d'autres enfants néandertaliens (tabl. 1).

\section{Face vestibulaire}

Comme il a été signalé plus haut la couronne est étroite avec des angles mésial et distal assez droits (fig. 4a). La surface de la dent est courbe comme chez tous les enfants néandertaliens, et la ligne cervicale est un petit peu déviée vers le côté mésial.

\section{Faces mésiale et distale}

À part une petite facette de contact de chaque côté, dans les deux perspectives (fig. 4b et 4c) la courbure de la face vestibulaire et la proéminence du tubercule lingual sont bien visibles.

\section{Face linguale}

La morphologie (fig. 4d) est un peu altérée par l'usure. Elle est concave, avec un tubercule lingual bien saillant, légèrement dévié vers le côté distal. La crête centrale et les crêtes marginales sont peu marquées. Rappelons qu'il n'a pas été signalé dans la littérature d'incisives centrales déciduales inférieures néandertaliennes avec une morphologie en pelle. 


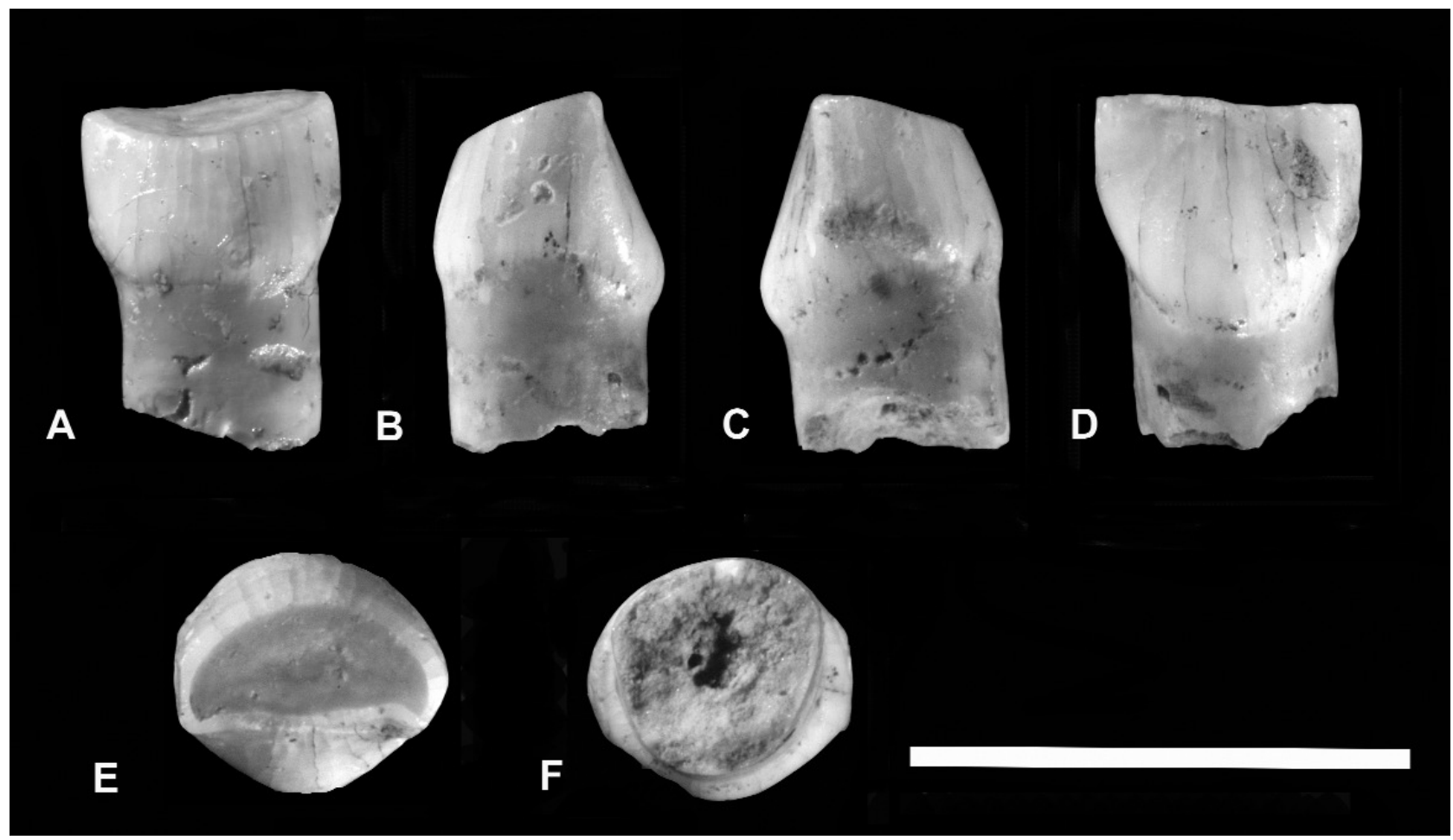

Figure 4 -Combe-Grenal 31 restaurée. $A$ : face vestibulaire. $B$ : face mésiale. $C$ : face distale. $D$ : face linguale. $E$ : face occlusale. $F$ : face apicale (cliché Ph. Jugie, Musée national de Préhistoire).

Figure 4 - Combe-Grenal 31 restored. A : labial face. $B$ : mesial face. $C$ : distal face. $D$ : lingual face. $E$ : occlusal face. $F$ : apical face (photo Ph. Jugie, Musée national de Préhistoire).

\section{Face occlusale}

La couronne est étroite mésio-distalement et les deux proéminences, celle de la face vestibulaire et, surtout, le tubercule lingual, se détachent nettement (fig. 4e).

Le bord occlusal est oblique vers le bas et vers la face vestibulaire, indiquant une occlusion de type I (Hillson 1996), et non « bout à bout » comme chez l'enfant de Roc-de-Marsal 1, probablement décédé plus jeune que celui représenté par Combe-Grenal 31. L'usure, très intense, a plus affecté le coté labial. Elle correspond au type 5 de Murphy (1959, modifié par Smith 1984), avec une ceinture d'émail tout autour et de la dentine secondaire au centre.

\section{Racine}

La racine est cassée (comme la prémolaire inférieure droite de la couche 39, Combe-Genal XV : Garralda et Vandermeersch 2000a), avec les bords érodés et sans signes de résorption. L'observation macroscopique laisse voir que toute la surface de la base de la racine est couverte d'une fine concrétion calcitique (fig. 4f), indiquant que la cassure s'était produite avant la fouille, au cours de processus taphonomiques qui eurent lieu pendant la formation de cette couche 60 .

\section{Âge au décès}

L'usure accentuée ainsi que la présence des deux facettes interproximales et l'absence de résorption de la partie préservée de la racine, permettent d'estimer un âge au décès de cet enfant d'environ 3 ans \pm 12 mois, en appliquant le schéma d'Ubelaker (1978) sur une série archéologique d'Amérindiens (et en ayant conscience de l'incertitude inhérente à ce type de collection), schéma très souvent utilisé par les paléoanthropologues.

\section{Assignation taxinomique}

En fonction de l'ensemble des données métriques (tabl. 1 et 2) et morphologiques présentées ci-dessus, il apparaît nettement que cette dent présente un diamètre mésio-distal plus élevé que la limite supérieure traduisant $99 \%$ (respectivement 5,13 et $4,93 \mathrm{~mm}$ ) de la variabilité, de nos deux échantillons $A$ et $B$ d'individus récents. II en est de même avec le diamètre vestibulo-lingual (limite supérieure $99 \%=$ 4,60 mm uniquement pour l'échantillon $B$, regroupant des individus européens, africains et asiatiques ; tabl. 2). Logiquement, cette dent montre donc une robustesse très importante et un indice de la couronne inférieur à l'actuel, conséquence de l'importance de sa longueur (qui est sous-estimée à cause des facettes interproximales et de l'attrition de la face occlusale). Comparée aux dimensions des Néandertaliens européens et proche-orientaux (tabl. 1 


\begin{tabular}{|c|c|c|c|c|}
\hline$\underline{\text { Individu }}$ & $\begin{array}{l}\text { D. mésio- } \\
\text { distal }\end{array}$ & $\begin{array}{l}\text { D. bucco- } \\
\text { lingual }\end{array}$ & $\begin{array}{c}\text { I. } \\
\text { couronne }\end{array}$ & $\begin{array}{c}1 . \\
\text { robustesse }\end{array}$ \\
\hline Combe-Grenal 31 & 5,22 & 4,70 & 90,04 & 24,53 \\
\hline Pech de l'Azè 1 & D 4,7 & D 4,8 & 102,13 & 22,56 \\
\hline (Tillier, 1979) & G 5,1 & G 4,5 & 88,24 & 22,95 \\
\hline $\begin{array}{c}\text { Chateauneuf } 2 \\
\text { (Tillier, 1979) }\end{array}$ & 4,7 & 4,0 & 85,11 & 18,8 \\
\hline $\begin{array}{c}\text { Krapina 1-45-45(1) } \\
\text { (Wolpoff, 1979) }\end{array}$ & 5,3 & 4,9 & 92,45 & 25,97 \\
\hline Kébara 1 & D 5,08 & D 5,0 & 98,43 & 25,4 \\
\hline (Smith \& Arensburg, 1977) & G 5,07 & G 5,05 & 99,61 & 25,6 \\
\hline $\begin{array}{c}\text { La Chaise } 14 \text { - Suard } \\
\text { (Tillier et Genet-Varcin, 1980) }\end{array}$ & 5,0 & 4,6 & 92,00 & 23,00 \\
\hline Roc de Marsal & D 4,7 & D 4,8 & 102,13 & 22,56 \\
\hline (Madre-Dupouy, 1992) & G 5,1 & G 4,5 & 88,24 & 22,95 \\
\hline $\begin{array}{c}\text { Shanidar } 7 \\
\text { (Senyurek, 1959) } \\
\end{array}$ & 5,2 & 4,9 & 94,23 & 25,48 \\
\hline $\begin{array}{l}\text { Zhou Kou Dian-B04 } \\
\text { (Weidenreich, 1937) }\end{array}$ & 4,3 & 3,6 & 83,72 & 15,48 \\
\hline Néandertaliens & $M=4,86$ & $M=4,66$ & $M=96,06$ & $M=22,65$ \\
\hline Spécimens du & $\mathrm{n}=14$ & $\mathrm{n}=14$ & $\mathrm{n}=14$ & $\mathrm{n}=14$ \\
\hline MIS $5 d$ au MIS 3 & $s=0,269$ & $s=0,268$ & $\mathrm{~s}=7,257$ & $\mathrm{~s}=1,988$ \\
\hline A: populations modernes & $M=4,14$ & $M=3,89$ & & \\
\hline origines diverses & $n=329$ & $n=329$ & & \\
\hline (Maureille, 2001) & $s=0,382$ & $s=0,386$ & & \\
\hline B : groupe moderne & $M=3,97$ & $M=3,69$ & 92,87 & 14,67 \\
\hline (Maureille, base de données & $\mathrm{n}=54$ & $\mathrm{n}=58$ & $\mathrm{n}=54$ & $\mathrm{n}=54$ \\
\hline pers.) & $s=0,359$ & $s=0,341$ & $s=7,905$ & $s=2,448$ \\
\hline
\end{tabular}

Tableau 1- Mesures (en $\mathrm{mm}$ ) et indices de Combe-Grenal 31, d'autres fossiles et des séries de comparaison. $M=$ moyenne, $n=$ effectif, $s=$ écart-type.

Table 1-Dimensions (in $\mathrm{mm}$ ) and indexes of Combe-Grenal 31, of some other fossils and of the comparison series. $M=$ mean, $n=$ sample size, $s$ = standart deviation.

\begin{tabular}{|c|c|}
\hline Echantillons & Constitution \\
\hline A : populations modernes & $\begin{array}{c}\text { Jordanie }(n=139) \text {, Amérique du Nord }(n \\
=177) \text {, Moyen-Orient }(n=15)\end{array}$ \\
\hline B : échantillon moderne & $\begin{array}{c}\text { Spitalfieds, Poundburry, Asie (Bengal), } \\
\text { Afrique }\end{array}$ \\
\hline $\begin{array}{l}\text { Néandertaliens, fossiles du stade isotopique } \\
\qquad 5 \text { d à } 3\end{array}$ & $\begin{array}{l}\text { Roc-de-Marsal 1, Le Portel n²1,30,22, } \\
\text { La Ferrassie 8, Pech-de-l'Azé 1, } \\
\text { Shanidar 7, Kébara 1, Château-neuf-sur- } \\
\text { Charente 2, Bau-de-l'Aubesier } 8\end{array}$ \\
\hline Pré-néandertaliens & $\begin{array}{l}\text { Krapina P1-45-45(1), } \\
\text { La Chaise Suard } 14\end{array}$ \\
\hline $\begin{array}{c}\text { Fossiles du Paléolithique moyen ou du Middle } \\
\text { Stone Age non rapportés à la lignée } \\
\text { néandertalienne }\end{array}$ & $\begin{array}{l}\text { Qafzeh 10, Skhul 1, Die Kelders Cave } \\
\text { AP6290 }\end{array}$ \\
\hline
\end{tabular}

Tableau 2 - Échantillons utilisés pour les comparaisons et l'analyse bivariée.

Table 2 - Samples used for the comparisons and bivariate analysis.

et 2) et aux deux fossiles européens mis au jour dans des niveaux très probablement antérieurs au stade isotopique 5e (Krapina et La-Chaise/Suard 14), Combe-Grenal 31 présente des dimensions et des proportions nettement plus proches de ces derniers que de fossiles plus récents. Pour expliquer ces résultats, on retrouve à nouveau la forte contribution du diamètre mésio-distal de la couronne.

Nous considérerons donc que cette incisive centrale déciduale inférieure s'intègre bien au sein de la variabilité de la lignée néandertalienne, bien qu'il s'agisse d'une dent dont les traits morphologiques et métriques sont peu discriminants.

\section{3 - Étude bivariée des diamètres coronaires de Combe-Grenal 31}

Pour tenter de mieux visualiser ces différences, nous avons réalisé un graphique bidimensionnel en fonction du diamètre mésio-distal et du diamètre vestibulo-lingual de la couronne (fig. 5). Pour les fossiles, toutes les dents disponibles ont été intégrées dans l'analyse, aussi bien les dents droites que les gauches d'un même sujet (tabl. 2). Nous pensons que cette démarche est légitime en raison de la faiblesse de l'effectif fossile, et du fait que nous tentons d'apprécier au mieux une variabilité métrique. Nous avons déjà signalé (Garralda et al. 2008), que les dents droite et gau- 


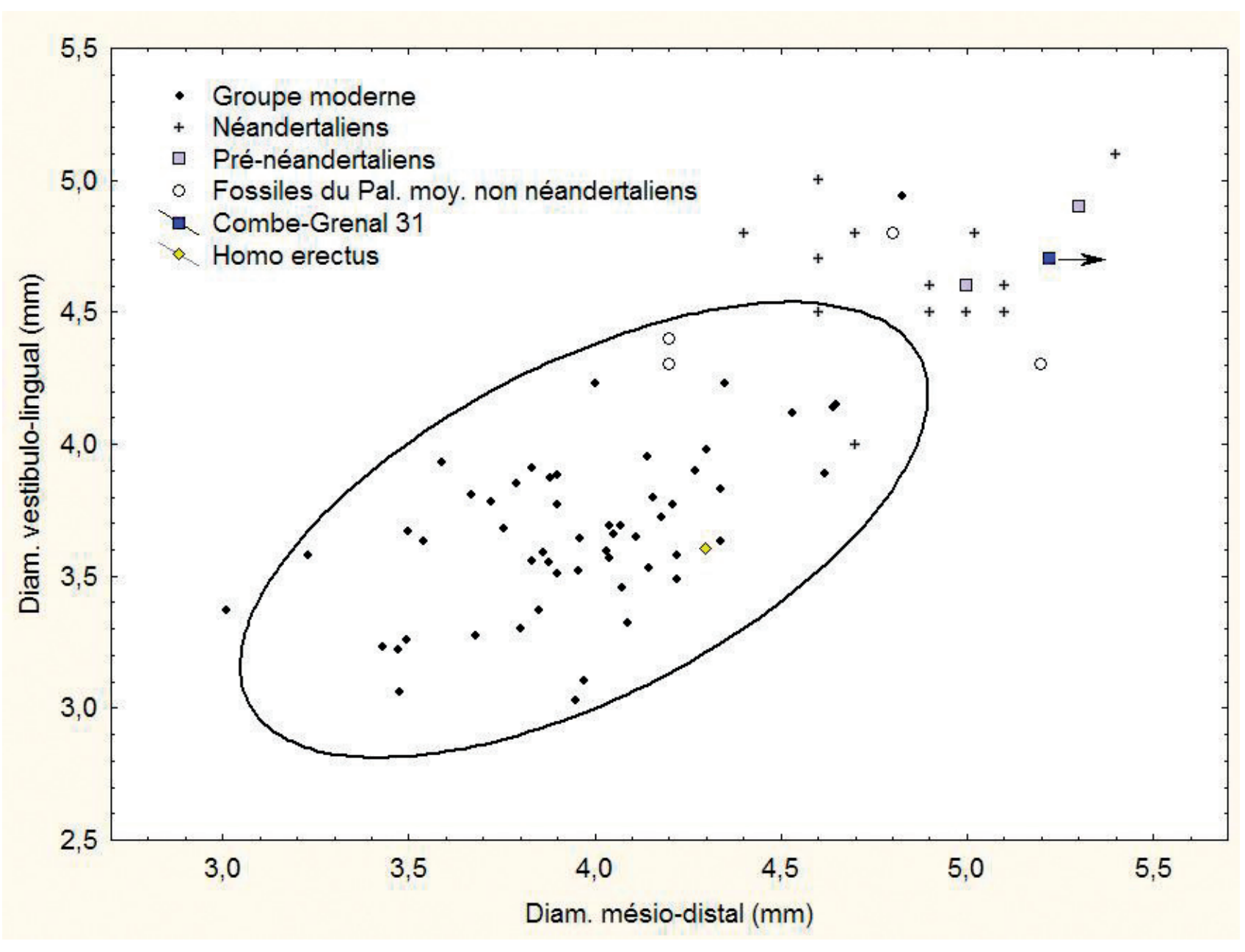

Figure 5 - Ellipse équiprobable (95 \% de la variabilité) réalisée à partir de M81 et M81(1) et position de Combe-Grenal 31 (carré bleu foncé) relativement à des groupes d'Hommes actuels (ronds noirs), un H. erectus (losange jaune), des Prénéandertaliens (carrés bleu clair), des Néandertaliens (croix) et d'autres fossiles du Paléolithique moyen (ronds blancs). Échantillons d'après Maureille 2001.

Figure 5 - Bivariate scatter plots (95\% of the variability) based on M81 and M81(1) and position of Combe-Grenal 31 (dark blue square) in relation groups of extant humans (black dots), one H. erectus (yellow rhomb), Pre-neandertals (light blue squares), Neandertals (crosses) and some other Middle Paleolithic fossils. Samples from Maureille 2001.

che d'un même sujet ne sont pas totalement similaires comme nous l'observons aussi dans le tableau 1. Nous avons décidé de rassembler dans un même groupe, les fossiles du Paléolithique moyen ou du Middle Stone Age qui n'ont jamais été supposés être membres de la lignée néandertalienne. Enfin, nous intégrons dans l'analyse l'unique incisive centrale déciduale inférieure rapportée à un Homo erectus, celui de Zhou Kou Dien B04 (Weidenreich 1937). Etant donné les effectifs de nos échantillons, I'unique ellipse représentée est celle du «B : groupe moderne » des tableaux 1 et 2 , et symbolise l'aire de $95 \%$ de sa variabilité.

Les sujets rapportés à ce dernier groupe se répartissent de façon assez homogène. Notons toutefois qu'un de ces individus a une incisive déciduale dont les dimensions sont très importantes et, par conséquent, il se trouve nettement exclu de $95 \%$ de la variabilité de son groupe. Les Néandertaliens des stades isotopiques $5 d$ à 3 présentent des dents aux diamètres nettement supérieurs à ceux des dents du groupe moderne et une longueur importante relativement à leur largeur. Les deux pré-néandertaliens se situent au niveau des dents néandertaliennes les plus longues. Il en est de même pour Combe-Grenal 31. Les fossiles du Pléistocène supérieur non rapportés à la lignée néandertalienne sont parfois exclus, parfois inclus au sein de la variabilité du groupe moderne. Enfin, I'unique Homo erectus se caractérise par une dent dont la couronne montre de petites dimensions.

Relativement à tous ces individus, Combe-Grenal 31 se caractérise donc par une couronne présentant des diamètres coronaires élevés à très élevés. 


\section{Conclusions}

Nous ne disposons pas de données pour discuter le ou les processus taphonomiques qui ont favorisé la conservation dans la couche 60 de Combe-Grenal de cette dent malheureusement incomplète, une incisive déciduale centrale inférieure droite d'un enfant de 3-4 ans (selon la variabilité actuelle).

Combe-Grenal 31 n'est pas un fossile très « discriminant 》 d'autant qu'il s'agit d'une dent usée et incomplète. Mais les données morphologiques (cingulum et saillie des crêtes linguales) et surtout métriques de CG31 s'intègrent mieux au sein de la variabilité des membres de la lignée néandertalienne que des Hommes anatomiquement modernes. En ce qui concerne les diamètres de la couronne dentaire, CG31 se situe à proximité des deux pré-néandertaliens des stades isotopiques $6-5$ e (Suard, Krapina). Au sein de leur lignée (et donc de fossiles plus récents des stades $5 \mathrm{~d}$, 4 et 3 : Roc-de-Marsal 1, Pech-de-l'Azé 1, Châteauneuf 2, Shanidar 7 et Kébara 1), ces derniers se caractérisent par une longueur importante relativement à la largeur de la couronne. Notons aussi que la variabilité de la lignée néandertalienne est importante, bien que nettement distincte de celle des Hommes modernes, et que s'y intègrent quelques sujets récents ou du Pléistocène supérieur.

Par conséquent, nous proposons I'hypothèse que le fossile Combe-Grenal 31 soit rapporté à la lignée néandertalienne.

Si l'étude de l'industrie lithique rapproche cette couche de techno-complexes proches du Pech-de-l'Azé II et de Combe-Brune 3 (entre 117 et $205 \mathrm{ka}$ ) le cortège faunique la situe avec fiabilité à la fin du stade isotopique 6 (entre 140 et $127 \mathrm{ka}$ ). La concordance des données technologiques et paléontologiques nous amène à dater cette couche 60 autour de $130 \mathrm{ka}$. Considérant l'ensemble de ces données, l'ancienneté de la couche 60 fait que ce nouveau vestige, Combe-Grenal Hominid 31, est actuellement le plus ancien fossile humain d'Aquitaine représentant un groupe de Pré-Néandertaliens qui habita cette région pendant le dernier tiers du stade isotopique 6.

Le terme d'Aquitaine a donc été pris ici au sens administratif de la région Aquitaine. Si on I'utilise dans un sens géologique ou géographique (Bassin aquitain), le reste humain du site de Pradayrol dans le Lot représente alors le fossile humain le plus ancien de ce territoire car provenant de niveaux mis en place antérieurement au stade isotopique 6 (Séronie-Vivien et Tillier 2002).

\section{Remerciements}

Nous tenons à remercier le Ministère de l'Education d'Espagne pour l'attribution à l'un de nous (M.-D.G.) d'une bourse de Recherche pendant trois mois en 2009 au sein de l'UMR PACEA (Campus de Bordeaux), ainsi que le projet "Transitions : d'une société à l'autre, processus d'adaptation et de peuplements ", convention 20051403003AB de la Région Aquitaine.
Nous exprimons notre reconnaissance aussi aux personnes et Institutions qui nous ont permis d'étudier les fossiles : J.-J. Cleyet-Merle (Musée national de Préhistoire, France), H. et M.-A. de Lumley (Institut de Paléontologie Humaine, Paris), Ph. Mennecier (Musée de l'Homme, Paris), R. Kruszinsky et C. B. Stringer (Natural History Museum, London), J. Radovcic (Hrvatski Prirodoslovni Muzej, Zagreb), A. et M. E. Segre (Instituto de Paleontologia Umana, Roma), G. Manzi (Universitá La Sapienza, Roma), N. Conard (Université de Tübingen).

Nous remercions également $\mathrm{Ph}$. Jugie, photographe au Musée national de Préhistoire des Eyzies-de-Tayac, pour son aide dans notre recherche iconographique, $D$. Faunières et $M$. Boucharat qui ont restauré la dent, et Françoise Delpech et Aurore Schmitt pour leurs commentaires et leur aide pour améliorer la qualité de cet article.

\section{Références bibliographiques}

BLACKWELL B., SCHWARCZ H.P. et DEBÉNATH A. 1983 Absolute dating of hominids and Paleolithic artifacts of the cave La Chaise-de-Vouthon (Charente), France. Journal of Archaeological Science, 10, p. 493-513.

BOËDA E. 1991 - La conception trifaciale d'un nouveau mode de taille paléolithique. In : E. Bonifay, et B. Vandermeersch (éd.) Les premiers Européens. Actes du $114^{\circ}$ Congrès national des Sociétés savantes (Paris, 39 avril 1989). Paris, C.T.H.S., p. 251-263, ill.

BORDES F. 1955 - La stratigraphie de la grotte de CombeGrenal (Dordogne). Note préliminaire. Bulletins de la Société Préhistorique Française, 52, p. 426-429.

BORDES F. 1961 - Typologie du Paléolithique inférieur et moyen. 2 vol., Bordeaux : Delmas, 85 p., ill., pl.

BORDES F. 1966 - Acheulean culture in South-West France. Studies in Prehistory Robert Bruce Foote Memorial volume, Calcutta, p. 49-59, 2 fig., 1 carte.

BORDES F. 1971 - Observations sur l'Acheuléen des grottes en Dordogne. Munibe, 1, p. 5-24.

BORDES F. 1972 - A tale of Two Caves. Harper \& Row, New York, 169 p., ill.

BORDES F. et PRAT F. 1965 - Observations sur les faunes du Riss et du Würm en Dordogne. L'Anthropologie, 69, p. 31-46.

BORDES F., LAVILLE H., de LUMLEY H., MISKOWSKY J.C., PAQUERAU M.-M., PILLARD B., PRAT F. et RENAULTMISKOWSKY J. 1972 - Le Würmien II. Tentative de corrélations entre le Languedoc méditerranéen (l'Hortus) et le Périgord (Combe-Grenal). Études Quaternaires, 1, p. 353362. 
BORDES F., LAVILLE H. et PAQUERAU, M.-M. 1966 Observations sur le Pléistocène supérieur du gisement de Combe-Grenal (Dordogne). Actes de la Société Linnéenne de Bordeaux (série B), p. 3-19.

BOURGUIGNON L. et TURQ A. 2003 - Une chaîne opératoire de débitage discoïde sur éclat du Moustérien à denticulés aquitain : les exemples de Champ de Bossuet et de Combe-Grenal c.14. In : M. Peresani (Ed.), Discoid lithic technology - Advances and implications. BAR International Series 286 Oxford.

BOWMAN S. E. J. et SIEVEKING J. D. J. 1983 Thermoluminescence dating of burnt flint from CombeGrenal. PACT, 9, p. 253-268.

BRENET M., FOLGADO M., LENOBLE A., BERTRAN P., VIEILLEVIGNE E., GUIBERT P. 2008 - Interprétation de la variabilité technologique du Paléolithique moyen ancien du Bergeracois : Cantalouette 1 et Combe Brune 3 (Creysse, Dordogne). Contexte géoarchéologique et chronologique, analyse techno-économique. Bulletins de la Societé Préhistorique Francaise, mém. XLVII, p. 57-82.

CHASE P. G. 1983 - The use of animal resources in the Mousterian of Combe-Genal, France. Ph. D. Dissertation. Univ. of Arizona.

CHASE P. G. 1986a - The hunters of Combe-Grenal : Approaches to Middle Paleolithic subsistence in Europe. British Archeological Reports, International series 286, p. 1-224

CHASE P. G. 1986b - Relationships between Mousterian lithic and faunal assemblages at Combe-Grenal, France. Current Anthropology, 27, p. 69-71.

CHASE P. G. 1989 - How different was Middle Paleolithic subsistence? A zooarchaeological perspective. In : Mellars P. et Stringer C. (Eds.), Behavioural and Biological Perspectives on the Origins of Modern Humans. Edinburgh University Press Edinburgh, p. 321-337.

DELPECH F. et PRAT F. 1980 - Les grands mammifères pléistocènes du Sud-Ouest de la France. In : Problèmes de stratigraphie quaternaire en France et dans les pays limitrophes. Suppl. Bull. Ass. Fr. Et. Quat., n. s., 1, p. 268-297, 3 fig., 1 tabl.

DELPECH, F. et PRAT, F. 1995. Nouvelles observations sur les faunes acheuléennes de Combe-Grenal (Domme, Dordogne). Paléo, 7, p. 123-137.

DELPECH F., GENESTE J.-M., RIGAUD J.-Ph. et TEXIER J.-P. 1995 - Les industries antérieures à la dernière glaciation en Aquitaine septentrionale : chronologie, paléoenvironnements, technologie, typologie et économie de subsistance. Paléo, supplément $n^{\circ} 1, \mathrm{p}$ 133-163, 11 fig., 3 tabl.
DIBBLE H. L., McPHERRON S. P., SANDGATE D., GOLDBERG P., TURQ A. et LENOIR M. 2009 - Context, curation, and bias : an evaluation of the Middle Paleolithic collections of Combe-Grenal (France). Journal of Archaeological Science 36, p. 2540-2550.

DONARD E. 1982 - Recherches sur les Léporinés quaternaires (Pléistocène moyen et supérieur, Holocène). Thèse de Doctorat en Géologie du Quaternaire et Préhistoire, Université Bordeaux 1, n¹764, 161 p. de texte, 89 tabl., 46 pl.

FAIVRE, J.-Ph. 2008 - Organisation techno-économique des systèmes de production dans le Paléolithique moyen récent du Nord-est Aquitain : Combe-Grenal et les Fieux. Thèse de l'Université Bordeaux 1, spécialité : Préhistoire et Géologie du Quaternaire, n 3597, p. 553, ill.

FERNANDEZ P., GUADELLI J.-L. et FOSSE Ph. 2006 Applying dynamics and comparing life tables for Pleistocène Equidae in anthropic (Bau de l'Aubesier, Combe-Grenal) and carnivore (Fouvent) contexts with modern feral horse populations (Akagera, Pryor Mountain). Journal of Archaeological Science, 33, p. 176-184.

GARRALDA M.-D. 2008 - Les Néandertaliens : d'autres gestes envers les défunts. In : B. Vandermeersch, J.J.Cleyet-Merle, J. Jaubert, B. Maureille et A. Turq (Éds), Première humanité, gestes funéraires des Néandertaliens. Paris : Réunion des Musées Nationaux, p. 42-51.

GARRALDA M.-D. et VANDERMEERSCH B. 1997 - Los Neandertales de Combe-Grenal (Dordogne, Francia). In : "Senderos de la Evolución Humana. Estudios en Homenaje a Phillip V. Tobias ». Volumen especial de la revista « Ludus Vitalis » (Mexico), p. 129-144

GARRALDA M.-D. et VANDERMEERSCH B. 2000a - Les Néandertaliens de Combe-Grenal (Domme, France). Paléo 12, p. 213-259.

GARRALDA M.-D. et VANDERMEERSCH B. 2000b - Los Neandertales de Combe-Grenal: Aspectos Bio-culturales de su dentición. In : T. A. Varela (Ed.), Investigaciones en Biodiversidad humana. Universidad de Santiago de Compostela, p. 231-237.

GARRALDA M.-D. et VANDERMEERSCH B. 2007 - $i$ Escorbuto en los Neandertales ? Posibles casos en Combe-Grenal (Domme, Francia). VELEIA Volumen Homenaje al Prof. I. Barandiarán, 24-25, p. 385-395.

GARRALDA M.-D., GIACOBINI G. et VANDERMEERSCH B. 2003 - Huellas de manipulación intencional en dos Neandertales de Combe-Grenal (Domme, Francia). In, M. Campo et F. Robles (Eds.), ¿ Dónde estamos ? Pasado, presente y futuro de la Paleopatología. Univ. Autónoma de Madrid y Asoc. Esp. de Paleopatología, p. 139-147. 
GARRALDA M.-D., GIACOBINI G. et VANDERMEERSCH B. 2005 - Cut marks on the Neandertals from CombeGrenal and Marillac (France). A SEM analysis. Anthropologie, XLIII/2-3, p. 251-271.

GARRALDA M.-D., MAUREILLE B., PAUTRAT Y. et VANDERMEERSCH B. 2008 - La molaire d'enfant Néandertalien de Genay (Côte-d'Or, France). Réflexions sur la variabilité dentaire des Néandertaliens. Paleo, 20, p. 89-99.

GENET-VARCIN E. 1982 - Vestiges humains du Würmien inférieur de Combe-Grenal, commune de Domme (Dordogne). Annales de Paléontologie (Vert.-Invert.), 68, p. 133-169.

GRÜN R., MELLARS P. et LAVILLE H. 1991 - ESR Chronology of a 100000 year archaeological sequence at Pech-de-l'Azé II, France. Antiquity, 65, p. 544-551.

GUADELLI J.-L. 1987 - Contribution à l'étude des zoocénoses préhistoriques en Aquitaine Würm ancien et interstade würmien). Thèse de l'Université Bordeaux 1, spécialité : Préhistoire et Géologie du Quaternaire, $n^{\circ}$ ordre 148, p. 565 , fig., tabl.

GUADELLI J.-L. 1996 - Les Cerfs du Würm ancien en Aquitaine. Paléo, 8, p 99-108.

GUADELLI J.-L. et LAVILLE H. 1990 - L'environnement climatique de la fin du Moustérien à Combe-Grenal et à Camiac. Confrontation des données naturalistes et implications. In : C. Farizy (Ed.), Paléolithique moyen récent et Paléolithique supérieur ancien en Europe. Mém. du Musée de Préhistoire d'lle de France, p. 43-48.

GUIBERT P., BECHTEL F., BOURGUIGNON L., BRENET M., COUCHOUD I., DELAGNES A., DELPECH F., DETRAIN L., DUTTINE M., FOLGADO M., JAUBERT J., LAHAYE C., LENOIR M., MAUREILLE B., TEXIER J.-P., TURQ A., VIEILLEVIGNE E., VILLENEUVE G. 2008 - Une base de données pour la chronologie du Paléolithique moyen dans le SudOuest de la France. Bulletin de la Société Préhistorique Française, mém. XLVII, p. 19-40.

GUICHARD J. 1965 - Un faciès original de l'Acheuléen : Cantalouette (commune de Creysse, Dordogne). L'Anthropologie, 69, p. 413-464.

GUICHARD J. et GUICHARD, G. 1966 - A propos d'un site Acheuléen du Bergeracois (Les Pendus, commune de Creysse) : bifaces-hachereaux et hachereaux sur éclat, aperçu typologique. Actes de la Société linnéenne de Bordeaux, série B, 103, 14 p.

HILLSON S. 1996 - Dental Anthropology. Cambridge Univ. Press, 373 p., ill.
HISCOCK P. et CLARKSON C. 2007 - Retouched notches at Combe-Grenal (France) and the reduction hypothesis. American Antiquity, 72, p. 176-190.

JAUBERT J. 1999 - Chasseurs et artisans du Moustérien. La Maison des Roches, Paris, 152 p.

LAQUAY G. 1981 - Recherches sur les faunes du Würm I en Périgord. Thèse de I'Université Bordeaux 1, spécialité Préhistoire et Géologie du Quaternaire, $n^{\circ}$ ordre 1596, 2 tomes, 429 p., tabl., pl.

LARTET E. et CHRISTY H. 1864 - Cavernes du Périgord. Revue Archéologique, 1, p. 233-267.

LAVILLE H. 1969a - Paléoclimatologie du Würm ancien en Périgord : données sédimentologiques. Proc. VIIIth. INQUA Congress, 1, p. 513-518.

LAVILLE H. 1969b - The relative position of Mousterian industries in the climatic chronology of the early Würm in the Perigord. World Archaeology, 4, p. 323-339.

LAVILLE H. 1975 - Climatologie et chronologie du Paléolithique en Périgord. Études Quaternaires, mém. 4, 423 p., ill.

LAVILLE H., RAYNAL J.-P. et TEXIER J.-P. 1984 Interglaciaire ou déjà glaciaire ? Bulletins de la Société Préhistorique Française, 81, p. 8-11.

LE MORT F. 1989 - Traces de décharnement sur les ossements néandertaliens de Combe-Grenal. Bulletins de la Société Préhistorique Française, 86, p. 79-87.

LENOIR M. 1986 - Un mode d'obtention de la retouche « Quina " dans le Moustérien de Combe-Grenal (Domme, Dordogne). Bulletins de la Société d'Anthropologie du Sud-Ouest, 21, p. 13-160.

LEVINE M. A. 1983 - Mortality models and the interpretation of horse population structure. In : G.N. Bailey (Ed.) Hunter-Gatherer Economy in Prehistory : a European Perspective. Cambridge Univ. Press, Cambridge, p. 23-46.

MADRE-DUPOUY M. 1992 - L'enfant de Roc-de-Marsal. Étude analytique et comparative. Cahiers de Paléoanthropologie, Paris : éds. du CNRS, 299 p., ill.

MARQUET J-C. 1989 - Paléoenvironnement et chronologie des sites du domaine atlantique français d'âge pléistocène moyen et supérieur d'après l'étude des Rongeurs. Thèse de Doctorat d'Etat ès Sciences naturelles, Université de Bourgogne, 637 p., 187 fig., 208 tabl., 6 pl.

MARTIN R. et SALLER K. 1957 - Lehrbuch der Anthropologie. Gustav Fischer Verlag, Stuttgart, 12211477 p., ill. 
MARTRAT B., GRIMALT J. O., LOPEZ-MARTINEZ C., CACHO I., SIERRO F. J., FLORES J. A., ZAHN R., CANALS M., CURTIS J. H., HODELL D. A. 2004 - Abrupt temperature changes in the Western Mediterranean over the past 250,000 years. Science, 306, p. 1762-1765.

MARTRAT B., GRIMALT J. O., SHACKLETON N. J., de ABREU L., HUTTERLI M., A., STOCKER T., F. 2007 - Four climate cycles of recurring deep and surface water destabilizations on the Iberian margin. Science, 317, p. 502-507.

MAUREILLE B. 2001 (inédit) - Variabilité dans le genre Homo : les mensurations de couronnes dentaires déciduales et permanentes. Synthèse de l'activité scientifique pour l'obtention de l'habilitation à diriger des recherches. Université Bordeaux 1, Laboratoire d'Anthropologie, 1, 168 p., ill.

MONGE J., MAUREILLE B. et MANN A. 2005 - La dentition humaine. In : O. Dutour, J.-J. Hublin et B. Vandermeersch (Éds.) Objets et Méthodes en Paléoanthropologie. Paris : éd. du CTHS, p. 103-151.

MONNIER G. F. 2006 - The lower/middle Paleolithic periodization in Western Europe : an evaluation. Current Anthropology, 47, p. 709-744.

MURPHY T. R. 1959 - Gradients of Dentine exposure in Human Tooth Attrition. American Journal Physical of Anthropology, 17, p. 179-185.

PANABIÈRES F. 1986 (inédit) - Étude techno-typologique des racloirs de la couche 35 de Combe-Grenal. Diplôme d'Études Approfondies en Préhistoire / Anthropologie. Université Bordeaux 1.

PAQUEREAU M.-M. 1970 - Flores et climats paléolithiques dans le sud-ouest de la France. Revue de Géographie Physique et de Géologie Dynamique, 12, p. 109-116.

PAQUEREAU M.-M. 1974-1975 - Le Würm ancien en Périgord. Étude palynologique. Quaternaria, 18, p. 67-159.

ROLLAND N. 1986 - Recent findings from la Micoque and other sites in south-western and mediterranean France : their bearing on the "Tayacian » problem and middle palaeolithic emergence. In : Barley G.N. and Callow P. (éd.), Stone Age Prehistory : studies in messing of Charles Mc Barney. Cambridge : Cambridge University press, p.121151, ill.

SENYUREK M. 1959 - A study of the deciduous teeth of the fossil Shanidar infant : a comparative study of the milk teeth of fossil men. Publ. Fac. Languages, History and Geograph . Univ. of Ankara, 128, 174 p.

SÉRONIE-VIVIEN M.-R. et TILLIER A.-M. 2002 - La dent humaine de Pradayrol (Caniac-du-Causse, Lot) dans son contexte stratigraphique et paléontologique. Paléo, 14, p. 257-264.
SMITH B. H. 1984 - Patterns of Molar Wear in HunterGatherers and Agriculturalists. American Journal of Physical Anthropology, 63, p. 39-56.

SMITH P. et ARENSBURG B. 1977 - A Mousterian skeleton from Kebara Cave. In : B. Arensburg et O. Bar-Yosef (Eds.) M. Stekelis Memorial volume. Jerusalem, The Israel exploration Society, p. 164-176.

STEELE T. 2003 - Using mortality profiles to infer behavior in the fossil record. Journal of Mammalogy, 84, p. 418-430.

STEELE T. 2004 - Variation in mortality profiles of red deer (Cervus elaphus) in Middle Palaeolithic assemblages from Western Europe. Journal of Osteoarchaeology, 14, p. 307320.

THIBAULT CI. 1970 - Recherches sur les terrains quaternaires du bassin de l'Adour. Thèse de I'Université Bordeaux 1, doctorat es sciences, 3 t., 385 p., ill.

THIÉBAUT C. 2006 - Le Moustérien à denticulés : variabilité ou diversité techno-économique ? Université de Provence, thèse de doctorat, spécialité préhistoire, 2 vol., 870 p., ill.

TILLIER A.-M. 1979 - La dentition de l'enfant moustérien Châteauneuf 2 découvert à l'abri de Hauteroche (Charente). L'Anthropologie, 83, p. 417-438.

TILLIER A.-M. et GENET VARCIN E. 1980 - La plus ancienne mandibule d'enfant découverte en France dans le gisement de La Chaise-de-Vouthon (abri Suard) en Charente. Z. Morph. Anthrop., 71, p. 196-214.

TURQ A. 1992 - Le Paléolithique inférieur et moyen entre les vallées de la Dordogne et du Lot. Bordeaux : Thèse de I'Université Bordeaux I, spécialité : Préhistoire et Géologie du Quaternaire, $n^{\circ}$ d'ordre : 778, 2 vol., 782 p., ill.

TURQ A. 2003 (inédit) - De la matière première lithique brute à la mise au jour de l'objet archéologique : propositions pour une meilleure exploitation du potentiel informatif du matériel lithique illustrées par quelques exemples du Paléolithique aquitain. Synthèse de l'activité scientifique pour l'obtention de l'habilitation à diriger des recherches. Université de Perpignan, 3 vols., 165 p.

UBELAKER D. 1978 - Human skeletal remains: excavation, analysis, interpretation. Chicago : Aldine, 41-67 p., ill.

VIEILLEVIGNE E., BOURGUIGNON L., ORTEGA I et GUIBERT P. 2008 - Analyse croisée des données chronologiques et des industries lithiques dans le grand sud-ouest de la France (OIS 10 à 3). Paléo, 20, p. 145-165.

WEIDENREICH F. 1937 - The dentition of the Sinanthropus pekinensis. A comparative odontography of the hominids. Palaeontologia Sinica, Peking, 111 p., pl.

WOLPOFF M. 1979 - The Krapina dental remains. American Journal of Physical Anthropology, 50, p. 67-114. 
